\title{
Congenital disorder of glycosylation type Ia (CDG-Ia): phenotypic spectrum of the $\mathrm{R} 141 \mathrm{H} / \mathrm{F} 119 \mathrm{~L}$ genotype
}

\author{
S Kjaergaard, M Schwartz, F Skovby
}

\begin{abstract}
Aims-To delineate common and variable features and outcome of children with congenital disorder of glycosylation type Ia (CDG-Ia) caused by the frequent R141H/F119L PMM2 genotype.
\end{abstract}

Methods-Clinical data on 25 patients (mean age 7.6 years, range 0-19) were analysed.

Results-All patients had an early presentation with severe feeding problems and failure to thrive, hypotonia, hepatic dysfunction, inverted nipples, and abnormal subcutaneous fat pads. Eighteen patients were hospitalised in the neonatal period. Developmental delay was obvious before age 6 months. During the first seven months mean standard deviation score (SDS) for weight and length decreased 2.7 $(\mathrm{SD}=2)$ and $2.4(\mathrm{SD}=2)$, respectively. Mental retardation, ataxia, muscular atrophy, and febrile seizures were consistent features after infancy. Variable features included pericardial effusions, afebrile seizures, and stroke like episodes. Computed tomography/magnetic resonance imaging of the brain was normal in two patients examined before 4 months of age, but 18 children examined after 3 months of age had cerebellar atrophy, and 10 children also had supratentorial atrophy. Subsequent imaging showed progression of the cerebellar and supratentorial atrophy in eight and four of 10 children, respectively. Mean head circumference SDS declined from zero to $-1.9 \mathrm{SD}$ from age 3 months to 5 years. Motor ability ranged from none to walking with a rolator, and vocabulary ranged from none to comprehensible speech. The overall mortality ascribed to CDG-Ia was $18 \%$.

Conclusion-Patients with the R141H/ F119L genotype have an early uniform presentation including severe failure to thrive, but their functional outcome is variable. This genotype may well cause clinical manifestations in the severe end of the spectrum of CDG-Ia.

(Arch Dis Child 2001;85:236-239)

Keywords: congenital disorder of glycosylation type Ia; phenotype; phosphomannomutase deficiency

Congenital disorders of glycosylation (CDG), formerly known as carbohydrate deficient glycoprotein syndromes, are a group of inherited multisystem disorders resulting from several genetic defects affecting the glycosylation of proteins. The most frequent CDG type Ia (CDG-Ia; OMIM 212065), caused by deficiency of phosphomannomutase (PMM) occurs worldwide. ${ }^{1}$ Among 58 different mutations in the encoding PMM2 gene, $\mathrm{R} 141 \mathrm{H}$ and F119L are the most common, ${ }^{23}$ and $86 \%$ of our patients are compound heterozygous for these mutations. ${ }^{4} \mathrm{~A}$ recent mutation update encompassing 23 countries revealed a total of 73 different genotypes, and the R141H/F119L genotype accounted for $27 \%$ of the 249 CDG-Ia patients, including ours. ${ }^{3}$ Most clinical data predate mutation analyses. To delineate common and variable features and outcome of patients with the R141H/F119L genotype, we analysed the clinical data from our large sample of CDG-Ia patients.

Patients and methods

PATIENTS

Twenty five patients (13 girls) with the R141H/F119L PMM2 genotype from 19 unrelated families of Danish origin were studied. The age range was $0-19$ years (mean 7.6 years). The diagnosis of CDG-Ia had been made by isoelectric focusing of serum transferrin, measurement of PMM activity in cultured fibroblasts, and mutation analysis. ${ }^{4}$ Two children who had died neonatally were diagnosed retrospectively by mutation analysis of DNA extracted from Guthrie cards following the diagnosis of a subsequent affected sib. The patient population included two sets of monozygotic and one set of dizygotic twins. Early manifestations of five patients have been reported previously. ${ }^{5}$

\section{METHODS}

All available clinical data were analysed, and additional pertinent information was obtained from interview of the parents. All patients except three were examined by at least one of the authors (SK and/or FS). Two sets of twins with same gender were typed for the microsatellite DNA markers D21S11, D21S1412, D21S1411, D18S535, S18S386, D13S258, D13S631, and HXPRT. Each pair of twins were identical for these markers, which made monozygosity highly probable (data not shown).

Measurements of length/height, weight, and head circumference were computed with standards for Danish children and expressed as standard deviation scores (SDS). ${ }^{67}$

Results

INCIDENCE

We know of 29 CDG-Ia patients in Denmark, 17 of whom were born between 1990 and 
Table 1 Clinical manifestations of CDG-Ia due to the R141H/F119L PMM2 genotype

\begin{tabular}{|c|c|c|}
\hline Manifestations & No./total tested & $\%$ \\
\hline \multicolumn{3}{|l|}{ Gastrointestinal symptoms } \\
\hline Feeding difficulties & $23 / 23$ & 100 \\
\hline Vomiting & $19 / 23$ & 83 \\
\hline Diarrhoea & $12 / 23$ & 52 \\
\hline Nasogastric tube/gastrostomy & $11 / 23$ & 48 \\
\hline \multicolumn{3}{|l|}{ Neurological manifestations } \\
\hline Cerebellar atrophy & $18 / 20$ & 90 \\
\hline Afebrile seizures & $13 / 23$ & 57 \\
\hline Febrile seizures & $21 / 23$ & 91 \\
\hline Stroke like episode & $12 / 23$ & 52 \\
\hline Hypotonia & $23 / 23$ & 100 \\
\hline Ataxia & $23 / 23$ & 100 \\
\hline Peripheral muscular atrophy & $23 / 23$ & 100 \\
\hline Psychomotor retardation & $23 / 23$ & 100 \\
\hline Absent tendon reflexes & $23 / 23$ & 100 \\
\hline Neurogenic hearing impairment & $3 / 9$ & 33 \\
\hline \multicolumn{3}{|l|}{ Hepatic dysfunction } \\
\hline Enlarged liver & $11 / 23$ & 48 \\
\hline Increased transaminases & $23 / 23$ & 100 \\
\hline Coagulation abnormalities & $23 / 23$ & 100 \\
\hline Episode of bleeding & $8 / 23$ & 35 \\
\hline Episode of ascites & $6 / 23$ & 26 \\
\hline Episode of thromboemboli & $1 / 23$ & 6 \\
\hline \multicolumn{3}{|l|}{ Cardiac involvement } \\
\hline Pericardial effusion & $10 / 19$ & 52 \\
\hline \multicolumn{3}{|l|}{ Dysmorphic features } \\
\hline $\begin{array}{l}\text { Inverted nipples } \\
\text { Inves }\end{array}$ & $23 / 23$ & 100 \\
\hline Abnormal fat distribution & $21 / 23$ & 91 \\
\hline Congenital joint restriction & $12 / 23$ & 53 \\
\hline Hernia/hydrocele/retentio testis & $10 / 12$ boys & 83 \\
\hline Esotropia & $23 / 23$ & 100 \\
\hline \multicolumn{3}{|l|}{ Skeletal abnormalities } \\
\hline Kyphoscoliosis & $11 / 23$ & 48 \\
\hline Thorax deformity & $20 / 23$ & 87 \\
\hline Fracture due to minimal trauma & $6 / 23$ & 26 \\
\hline
\end{tabular}

1998, corresponding to an incidence of 1 per 41000 newborns (all genotypes included). Twenty five of the 29 children had the R141H/ F119L genotype, corresponding to an incidence of 1 per 47000 newborns. In this calculation each set of identical twins was counted as one patient.

\section{CLINICAL PHENOTYPE}

Unless otherwise specified, the two children who died as neonates are excluded from the following analyses of clinical manifestations.

\section{NEONATAL COURSE}

Twenty two patients delivered at term had normal birth weights and lengths; mean SDS for weight and length were $-0.3(\mathrm{SD}=2)$ and 0 $(\mathrm{SD}=2)$, respectively. Three preterm neonates (one patient with a gestational age of 36 weeks and a set of twins with a gestational age of 35 weeks) had appropriate weight and length. Six children had Apgar scores of less than eight at one minute. These and 15 additional children were hospitalised as neonates because of cyanosis, respiratory distress, hypoglycaemia, dysmorphic features, oedema, lethargy, feeding problems, and/or failure to thrive.

Two children died as neonates. One patient developed thromboemboli of the abdominal aorta and common iliac artery following umbilical artery catheterisation; he died at 10 days of age during a cardiac catheterisation. Another newborn died as a result of rupture of the tentorium cerebelli.
GASTROINTESTINAL SYMPTOMS

All 23 patients had severe feeding problems in the first two years of life. They showed little interest in nursing or taking a bottle, were late to eat by spoon, and late to eat solid foods. Feeding difficulties prompted nasogastric tube feeding in 11 children and placement of a gastrostomy in one child. Failure to thrive was prominent in early life. At age 7 months mean SDS for weight was $-3.0(\mathrm{SD}=2)$, and mean SDS for length was $-2.4(\mathrm{SD}=2)$. Comprehensive longitudinal growth data will be reported separately. Vomiting and diarrhoea were prominent (table 1), especially during intercurrent infections.

\section{NEUROLOGICAL FINDINGS}

Head lag and poor visual fixation made developmental delay obvious in all 23 patients before 6 months of age. Hypotonia, ataxia, peripheral muscular atrophy, and lack of deep tendon reflexes were uniform findings. Computed tomography (CT) or MRI of the brain was normal in two patients examined before 4 months of age, but 18 children examined after 3 months of age had cerebellar atrophy. Ten children also had supratentorial atrophy (cortical and/or central). The two patients without cerebellar atrophy were each examined by CT only once, at 2 days and 4 months of age, respectively. In eight of 10 patients, subsequent imaging one month to seven years later showed progression of the cerebellar atrophy, and four patients had progression of supratentorial atrophy. The central nervous system was macroscopically normal in the two children who died as neonates. Measurements of head circumference were available from 22 children. Mean head circumference SDS declined from zero to -1.9 SD from age 3 months to 5 years. Febrile seizures were very frequent (table 1 ). The mean age at first event was 17 months (range 3-53 months). Afebrile seizures occurred in 13 children, eight of whom had recurrent convulsions. There was no preponderance of any type of seizure. The types observed included generalised seizures (tonic-clonic, atonic, and absence) and focal seizures (simple and complex partial). Three children suffered from recurrent migraine like headaches.

\section{STROKE LIKE EPISODES}

Stroke like episodes with hemiplegia and/or coma for more than 24 hours had occurred in $52 \%$ of the patients. Mean age at first event was 3.6 years (range $0.6-8.8$ years). The majority of such episodes appeared in association with fever and seizures. Six patients had more than one episode. CT of the brain performed during stroke like illnesses failed to reveal focal pathology $(n=5)$. The hemiplegia subsided over periods of two days to six months. One child had permanent loss of skills after a stroke like episode at age 10 .

\section{HEPATIC DYSFUNCTION}

Hepatomegaly (liver edge more than $3 \mathrm{~cm}$ below the right costal margin) was observed in $48 \%$ of the patients. All patients had had raised serum aminotransferases, and low prothrombin and/or 
antithrombin III. Serial measurements of serum aminotransferases showed improvement with increasing age, but impairment occurred during intercurrent infections (data not shown). All children had low concentrations of albumin, but ascites was observed only in six patients (table 1). Gastrointestinal, mucosal, or cutaneous bleedings were seen in 35\% of the patients, but none of these episodes was life threatening.

CARDIAC INVOLVEMENT

Echocardiography during the first year of life revealed pericardial effusions in 10 of 19 patients so examined (table 1). Surgical draining was necessary in four cases with compromise of ventricular function, and in one patient recurrent effusions led to pericardiectomy. The pericardial effusions of the remaining patients were stable or regressed spontaneously. No structural abnormality or cardiomyopathy was detected.

DYSMORPHIC FEATURES

All 23 children had inverted nipples in early childhood. At the most recent examination inverted nipples were still present in all but two patients, a 9 year old girl and a 15 year old boy. Subcutaneous suprapubic, perineal, and/or gluteal fat pads had been observed in $91 \%$ of the children. At the most recent examination such fat pads had disappeared in nine patients (age range 1.6-19.3 years).

Dysmorphic facial features were a uniform finding and included almond shaped, upslanting, palpebral fissures and a prominent maxilla with widely spaced permanent teeth. The ears appeared large, but ear length was below $+1 \mathrm{SD}$ in all but two cases $\left(\mathrm{n}=14\right.$, data not shown). ${ }^{8}$ The ears probably appeared large because of insufficient head growth and microcephaly.

Limited range of joint movement, especially of elbows and knees, was reported in $52 \%$ of the newborns. Physiotherapy improved the range of motion in the majority of these children. One patient had congenital clubfeet. The majority of the males had hydroceles, inguinal hernias, and/or non-descended testes (table 1).

\section{EYE AND EAR ABNORMALITIES}

All patients had esotropia. A systematic investigation of other eye abnormalities will be reported separately. Severe neurogenic hearing impairment was detected in three of nine patients tested for brain stem evoked potentials. Two of these children have affected sib(s) without hearing impairment. Eleven patients had had recurrent purulent otitis media, and three children had a conductive hearing loss caused by permanent perforation of the tympanic membrane.

SKELETAL ABNORMALITIES

Kyphoscoliosis was present in $48 \%$ of the patients above age 3. Pectus carinatum or excavatum was a constant finding after age 2 . Fractures caused by minimal trauma were observed in two boys and four girls, one of whom had had five fractures of large tubular bones.
THROMBOEMBOLIC EVENTS

Two patients had thromboemboli during the neonatal period. One child had an occlusion of a common iliac artery on the second day of life, but thrombolytic therapy restored blood flow. The other patient with thromboemboli of the abdominal aorta and a common iliac artery following umbilical artery catheterisation died neonatally.

ENDOCRINOLOGICAL FINDINGS

None of the girls had spontaneous onset of puberty $(n=3)$, and hormone analyses showed hypergonadotropic hypogonadism $(n=3)$. Ultrasonography showed hypoplastic internal genitalia $(n=2)$, and no oestradiol response was observed during a human menopausal gonadotropin stimulation test $(n=1$, Humegon, Organon, 75 IE intramuscularly once daily for 14 days). Oestradiol therapy (1 mg daily) induced development of breast tissue and pubic hair in two females.

FUNCTIONAL ABILITY

Fourteen patients were able to sit without support at the age of 20 months to 5 years. Five children above age 4 (range $4.3-9.9$ years) were unable to sit without support. Two patients walked with the aid of a rolator, and the majority were mobile in a wheelchair or a gait trainer. Two patients had not achieved ambulatory function at 9 years of age. All patients except four had to be fed. Only one patient was toilet trained. One patient had comprehensible speech, and 10 patients had an active vocabulary of 10-50 words comprehensible for other than close relatives. The passive vocabulary was generally better. Functional outcome was variable even among sibs and identical twins.

MORTALITY

Seven patients had died. One patient died at age 15 following a car accident, another died at 10 days of age during a cardiac catheterisation, and a third died as a newborn as a result of rupture of the tentorium cerebelli. Excluding these three patients, four patients had died from causes presumably related to CDG-Ia. The causes of death were pneumonia ( 4.3 and 6.0 years), septic shock (1.8 years), and unclassified infectious disease (9.9 years).

\section{Discussion}

The high incidence of CDG-Ia in Denmark, 1 per 41000 , and the predominance of the R141H/F119L PMM2 genotype are explained by a high carrier frequency of the $\mathrm{R} 141 \mathrm{H}$ mutation (1 in 60) in the Danish population and a founder effect of the F119L mutation in southern Scandinavia. ${ }^{9}{ }^{10}$ Although $\mathrm{R} 141 \mathrm{H}$ is present in $75 \%$ of white patients, homozygosity has never been observed. ${ }^{3}$ The lack of enzymatic activity of the recombinant mutant protein suggests that this genotype is incompatible with life, ${ }^{112}{ }^{12}$ and it explains the discrepancy between the expected frequency of CDG-Ia of 1 per 15000 , based on the carrier frequency of $\mathrm{R} 141 \mathrm{H}$ and the observed frequency of CDG-Ia. ${ }^{9}$ 
The clinical spectrum of CDG-Ia has expanded since the first description by Jaeken et al in $1980 .{ }^{13}$ Moderate to severe neurological impairment, variable dysmorphic features, and variable involvement of other organs are recognised now as common features, and a phenotype with only borderline cognitive dysfunction has been reported recently. ${ }^{14}$ Allelic heterogeneity among patients has hampered previous attempts to study the genotype-phenotype correlation, ${ }^{15} 16$ but the predominance of the $\mathrm{R} 141 \mathrm{H} / \mathrm{F} 119 \mathrm{~L}$ compound heterozygosity in our large series of CDG-Ia patients allowed us to study the phenotype and outcome of this particularly frequent PMM2 genotype.

The patients had an uniform early presentation with severe feeding problems, severe failure to thrive substantiated by a dramatic decline in SDS for weight and length, severe hypotonia, developmental delay obvious before age 6 months, hepatic dysfunction, inverted nipples, and subcutaneous fat pads. Pericardial effusion was a frequent finding in the first year of life, but rarely provoked symptoms. The striking initial presentation, often prompting hospitalisation in the neonatal period, should enable the clinician to make an early clinical diagnosis of CDG-Ia. The subcutaneous fat pads most often disappear with age, and the inverted nipples may become everted, obscuring the diagnosis in a minority of older children.

Cerebellar atrophy in CDG-Ia has been reported to have a neonatal onset. ${ }^{17}{ }^{18}$ Our imaging data document that atrophy of the cerebellum is not always present in newborn patients, and that the cerebellar and cerebral atrophy runs a progressive course in some patients. The decline in head circumference SDS reflects this progression.

Absence of puberty and hypergonadotropic hypogonadism are characteristic features in girls with CDG-Ia. Oestradiol secretion in response to exogenous gonadotropins has suggested biological inactivity of FSH. ${ }^{19}$ The lack of response in one of our patients confirms that primary ovarian failure may explain the absence of puberty. ${ }^{20}$

The distribution of clinical manifestations in our CDG-Ia population is close to that found in a compilation of Scandinavian patients prior to mutation analysis. ${ }^{21}$ Subsequently, the $\mathrm{R} 141 \mathrm{H} / \mathrm{F} 119 \mathrm{~L}$ genotype has in fact been shown to predominate in Scandinavia. ${ }^{3}$

Previous reports of functional ability in CDG-Ia patients have dealt with molecularly uncharacterised patients. ${ }^{1722}{ }^{23}$ Most were able to walk with support, and a few were able to walk without assistance. Most children could feed themselves and became toilet trained. In contrast, none of our patients were able to walk without support, only four managed to feed themselves, and only one was toilet trained. Although the functional outcome was variable, ranging from no ambulatory ability to walking with a rolator, and from no vocabulary to comprehensible speech, patients with $\mathrm{R} 141 \mathrm{H} /$ F119L probably represent the severe end of the clinical spectrum of CDG-Ia. This knowledge has implications for the management of the patients and for counselling of their families.
We are indebted to our patients and their families for willingness to participate in the study. We thank our colleagues Anna Berg, Alice Bernsen, Mette Brems, Karin Brostrøm, Niels Christian Christensen, Eva Fogh, Bente Hamborg-Petersen, Flemming Juul Hansen, Lars Kjærsgård Hansen, Karen KonstantinHansen, Kaj Lillquist, Jørgen Paulin, Vibeke Fauerholt Pedersen, Minna Bloch Petersen, and John Østergaard for providing clinical information and blood samples. We acknowledge Niels Erik Skakkebæk for investigations of the gonadal function of two patients and Jytte Bieber Nielsen for DNA marker anlysis of the twins. SK was supported by University of Copenhagen sis of the twins. SK was suppor
and Dagmar Marshall's Fond.

1 Van Schaftingen E, Jaeken J. Phosphomannomutase deficiency is a cause of carbohydrate-deficient glycoprotein syndrome type I. FEBS Lett 1995;377:318-20.

2 Matthijs G, Schollen E, Pardon E, et al. Mutations in PMM2, a phosphomannomutase gene on chromosome $16 \mathrm{p} 13$, in carbohydrate-deficient glycoprotein type I syndrome (Jaeken syndrome). Nat Genet 1997;16:88-92.

3 Matthijs G, Schollen E, Bjursell C, et al. Mutation update: mutations in PMM2 cause congenital disorders of glycosylation, type Ia (CDG-Ia). Hum Mutat 2000;16:38694.

4 Kjaergaard S, Skovby F, Schwartz M. Absence of homozygosity for predominant mutations in PMM2 in Danish patients with carbohydrate-deficient glycoprotein syndrome type 1. Eur F Hum Genet 1998;6:331-6.

5 Petersen MB, Brostrøm K, Stibler H, Skovby F. Early manifestations of the carbohydrate-deficient glycoprotein syndrome. F Pediatr 1993;122:66-70.

6 Andersen E, Hutchings B, Jansen J, Nyholm M. Heights and weights of Danish children. Ugeskr Laeger 1982;144: $1760-5$.

7 Jansen J. Measurements of head circumference at health examination of children. A multicentre study. Ugeskr Laeger 1982;144:3337-42.

8 Farkas LG. Anthropometry of the head and face in medicine. New York: Elsevier, 1981

9 Schollen E, Kjaergaard S, Legius E, et al. Lack of Hardy-Weinberg equilibrium for the most prevalent PMM2 mutation in CDG-Ia (congenital disorders of glycosylation type Ia). Eur f Hum Genet 2000;8:367-71.

10 Bjursell C, Wahlström J, Berg K, et al. Detailed mapping of the phosphomannomutase 2 (PMM2) gene and mutation detection enable improved analysis for Scandinavian CDG type I families. Eur f Hum Genet 1998;6:603-11.

11 Kjaergaard S, Skovby F, Schwartz M. Carbohydratedeficient glycoprotein syndrome type 1A: expression and characterisation of wild type and mutant PMM2 in E. coli. Eur F Hum Genet 1999;7:884-8.

12 Pirard M, Matthijs G, Heykants L, et al. Effect of mutations found in carbohydrate-deficient glycoprotein syndrome type IA on the activity of phosphomannomutase 2. FEBS Lett 1999;452:319-22.

13 Jaeken J, Vanderschueren-Lodeweyckx M, Casaer P, et al. Familial psychomotor retardation with markedly fluctuating serum prolactin, FSH and GH levels, partial TBGdeficiency, increased serum arylsulphatase A and increased CSF protein: a new syndrome? Pediatr Res 1980;14:179.

14 van Ommen $\mathrm{CH}$, Peters M, Barth PG, et al. Carbohydratedeficient glycoprotein syndrome type 1a: a variant phenotype with borderline cognitive dysfunction, cerebellar hypoplasia, and coagulation abnormalities. $f$ Pediatr 2000;136:400-3.

15 Imtiaz F, Worthington V, Champion M, et al. Genotypes and phenotypes of patients in the UK with carbohydratedeficient glycoprotein syndrome type 1 . F Inherit Metab Dis 2000;23:162-74.

16 de Lonlay P, Seta N, Barrot S, et al. A broad spectrum of clinical presentations in congenital disorders of glycosylation I: a series of 26 cases. 7 Med Genet 2001;38:14-19.

17 Jaeken J, Stibler H, Hagberg B, eds. The carbohydratedeficient glycoprotein syndrome: a new inherited multisystemic disease with severe nervous system involvement. Acta Paediatr Scand 1991;375(suppl):1-71.

18 Horslen SP, Clayton PT, Harding BN, et al. Olivopontocerebellar atrophy of neonatal onset and disialotransferrin developmental deficiency syndrome. Arch Dis Child 1991; 61:1027-31

19 de Zegher F, Jaeken J. Endocrinology of the carbohydratedeficient glycoprotein syndrome type 1 from birth through adolescense. Pediatr Res 1995;37:396-401.

20 Kristiansson B, Stibler H, Wide L. Gonadal function and glycoprotein hormones in the carbohydrate-deficient glycoprotein (CDG) syndrome. Acta Paediatr 1995;84:655-60.

21 Hagberg BA, Blennow G, Kristiansson B, Stibler H. Carbohydrate-deficient glycoprotein syndromes. In: Moser HW, ed. Handbook of clinical neurology. Amsterdam: Elsevier Science, 1996:623-37.

22 Stibler H, Blennow G, Kristiansson B, et al. Carbohydratedeficient glycoprotein syndrome: clinical expression in adults with a new metabolic disorder. 7 Neurol Neurosurg Psychiatry 1994;57:552-6.

23 Barone R, Pavone L, Fiumara A, et al. Developmental patterns and neuropsychological assessment in patients with carbohydrate-deficient glycoprotein syndrome type Ia (phosphomannomutase deficiency). Brain Develop 1999; 21:260-3. 